\title{
Silver nanoparticles synthesis from a pharmaceutically important medicinal plant Plumbago Zeylanica
}

\begin{abstract}
Purpose: In the Ayurvedic system of medicine, Plumbago zeylanica is one of the important medicinal plants which possess several pharmaceutical activities such as antiplasmodial, anti-microbial, antitumour, hepatoprotective, antifungal, antiinflammatory and anticancer activity. It contains several active constituents and the most important bioactive compound is plumbagin which is a naphthoquinone. Recently nanoparticles have gained its significance in the medical fields due to its higher surface area to volume ratio. In this study silver nanoparticles were synthesised from the in-vitro grown accession of Plumbago zeylanica.
\end{abstract}

Materials and methods: Aqueous extract of aerial part of Plumbago zeylanica accession number 524441 was analyzed for the presence of steroid, reducing sugar, flavonoids, alkaloids, tannin, terpenoids and saponins. Silver nanoparticles were synthesized by mixing the aqueous and silver nitrate solution for $24 \mathrm{hrs}$. Ultravioletvisible spectroscopy (UV-Vis) was used to detect the formation of silver nanoparticles and scanning electron microscopy (SEM) was used to check the shape of the synthesized nanoparticles.

Results: Present investigation deals with the qualitative analysis of phytocompounds present in the Plumbago zeylanica accession number 524441 was done using aqueous extract of the aerial part. It showed positive results for reducing sugar, terpenoid, steroid, flavonoid, glycoside and alkaloid. Further silver nanoparticles synthesis was done and synthesized nanoparticles were characterized by UV-VIS spectroscopy and SEM.

Conclusion: This is the first report on rapid and efficient synthesis of silver nanoparticles from the in-vitro grown accession of Plumbago zeylanica. It can be concluded that aerial part of Plumbago zeylanica can be a good source for the silver nanoparticles synthesis.

Keywords: plumbago zeylanica, silver nanoparticles, bioactive compounds, phytocompounds
Volume 3 Issue 5 - 2017

Arpita Roy, Navneeta Bharadvaja

Department of Biotechnology, Delhi Technological University, India

Correspondence: Navneeta Bharadvaja, Plant Biotechnology Laboratory, Delhi Technological University, New Delhi-I I0042, India, Email navneetab@dtu.co.in

Received: July 20, 2017| Published: August 14, 2017

\section{Introduction}

Plumbago zeylanica is a medicinal plant commonly called "White leadwort" in English and "Chitrak" in Sanskrit, it belonging to the Plumbaginaceae family. It's a perennial herb which is found in West Bengal, Uttar Pradesh and Maharashtra and also to some parts of South India. Most commonly used name persisted with the plant is chitraka. ${ }^{1}$ It having multi-purpose medicinal property and is most commonly used in the traditional medicinal system of India. The plants consist of various bioactive compounds like alkaloids, flavonoids, naphthoquinones, glycoside, saponins, steroids, triterpenoids, coumarins, phenolic compounds, tannins, carbohydrate, fixed oils, fats and proteins are present in different parts of the plant which have been reported to show anti-bacterial, anti-plasmodial, anti-tumour, hepatoprotective, central nervous system stimulatory activity, antifungal, anti-inflammatory, anti-hyperglycemic, anti-cancer and anti-atherosclerotic activity. ${ }^{2}$ Roots of $P$. zeylanica are long and slightly branched with very less secondary roots, having a smooth and unbroken texture. These roots are usually very strong having a bitter taste and a distinct odour with acrid. ${ }^{2}$ Medicinal properties of the plants are due to the presence of various phytocompounds. Qualitative analysis reveals the presence of different phytocompounds present in the plant.

In recent times nanotechnology is one of the fast growing fields. It mainly concerns to the synthesis and designing of nanomaterials within the range of $1-100 \mathrm{~nm}$. Nanoparticles exhibit new properties due to its specific characteristics such as size, morphology and distribution if compared to with the large particles of bulk material. Nanoparticles present a high surface to volume ratio with decreasing size. Generally, nanomaterials synthesized by using physical and chemical methods but the by-products from these methods are toxic in nature and the process is also costly. Chemically synthesized silver nanoparticles also showed poor antibacterial activity compared to biologically synthesized nanoparticles. This could be due to the protein coating of NPs obtained from the biological extract. ${ }^{3}$ To overcome this problem there is a need for environment-friendly methods of synthesis. Synthesis of nanoparticles using plant extract is an important branch. ${ }^{4}$ Several nanoparticles have been synthesized by this plant like silver, gold and copper oxide. Utilization of plant for nanoparticles synthesis 
can be advantageous over other biological processes as it eliminates the elaborate process of cell culture maintenance. Among several metal nanoparticles, silver nanoparticles gained special focus due to its stability, good conductivity and antimicrobial activity.

\section{Materials and methods}

Accessions number 524441 of Plumbago zeylanica was collected from NBPGR, New Delhi and maintained at the plant tissue culture laboratory of Department of Biotechnology, Delhi Technological University.

\section{Inoculation and incubation of explants}

The laminar air flow chamber was properly surface sterilized with alcohol and UV lights for 30minutes. The explants were trimmed to a suitable size of about $2 \mathrm{~cm}$ by keeping it in sterile Petri-dishes. Then a cut was given on both basal and top portion to remove the undesirable or dead portion. The forceps were rinsed in $70 \%$ ethanol and were flamed and then kept for some time to get cool. Each explant was then aseptically inoculated on the MS medium containing $1 \mathrm{mg} / 1 \mathrm{BAP}$ in an erect position with long forceps without touching the rim of the culture tube. Inoculated culture tubes were incubated in the culture room at $25 \pm 2^{\circ} \mathrm{C}$, with a light intensity of $2500 \mathrm{lux}$ and a photoperiod of 16 hour light, 8hour dark and $65 \%$ humidity.

\section{Preparation of aqueous plant extract}

In-vitro grown plant materials were dried for seven days. Dried plant material was used for the preparation of extract. $100 \mathrm{mg}$ of dried plant material mixed with $10 \mathrm{ml}$ of mili-Q water and boiled for 15 minutes. Then the plant extract was filtered through Whatman No.-1 filter paper and transferred into autoclaved vials and stored at $4{ }^{\circ} \mathrm{C}$ for further analysis.

\section{Analysis of phytochemicals}

Terpenoid test (salkowski test): $100 \mu 1$ of plant extract was mixed with $2 \mathrm{ml}$ of chloroform and $3 \mathrm{ml}$ of concentrated sulphuric acid to form a layer. A reddish brown color at interface shows the presence of terpenoid and triterpenoids.

Steroid test (salkowski test): $100 \mu$ l of plant extract was dissolved in $2 \mathrm{ml}$ of chloroform. Sulphuric acid was carefully added to form a lower layer. A reddish brown color at interface shows the presence of steroidal ring.

Saponin test (foam test): Add $100 \mathrm{mg}$ of powdered plant material to $10 \mathrm{ml}$ of distilled water. Mixture was heated and observed for persistent froth. Formation of froth shows the presence of saponins.

Flavonoid test (NaOH test): $100 \mu$ l of plant extract was treated with few drops of sodium hydroxide solution. Formation of intense yellow colour, which becomes colourless on the addition of dilute HCL, indicates the presence of flavanoids.

Tannin test $\left(\mathrm{FeCl}_{3}\right.$ test): $100 \mu \mathrm{l}$ of plant extract was treated with few drops of freshly prepared $6 \% \mathrm{FeCl}_{3}$. Green colour formation indicates the tannin presence.

Glycosides test (fehling's test): Equal volume of Fehling A and Fehling B reagents were mixed together and $2 \mathrm{ml}$ of it was added to the $100 \mu \mathrm{l}$ of plant extract and gently boiled. Formation of brick red precipitate at the bottom of the test tube indicates the reducing sugars presence.
Alkaloids (mayer's reagent): One milli-liter aqueous extract was taken in test tube and $2 \mathrm{ml}$ of $2 \mathrm{NHCL}$ was added to it then solution was shaken vigorously to mix and kept for 10minutes. Few drops of Mayer's reagent were added to it. Formation of creamy colour precipitation indicates the presence of alkaloids.

\section{Synthesis of silver nanoparticles}

Preparation of silver nitrate solution: Silver nitrate $\left(\mathrm{AgNO}_{3}\right)$ was collected from Fisher Scientific. For the preparation of $1 \mathrm{mM} \mathrm{AgNO}_{3}$ solution, $16.987 \mathrm{mg}$ of $\mathrm{AgNO}_{3}$ was added to $100 \mathrm{ml}$ of mili-Q water and mixed. Solution was stored in flask covered with aluminium foil.

Synthesis of silver nanoparticles: Potential of biosynthesis of silver nanoparticles of Plumbago zeylanica was tested by using leaf extract. Leaves of $P$. zeylanica were taken and dried under room temperature for 5 days. Then the dried leaves were crushed using pestle and mortar. The crushed material was then boiled in $10 \mathrm{ml}$ of distilled water. Then the extract was left to cool at room temperature. The cooled extract was filtered into fresh vials. $8.5 \mathrm{mg}$ of silver nitrate was dissolved in $50 \mathrm{ml}$ of distilled water. The extract was then added in the ratio of 1:10. Solution was mixed well and kept in incubator shaker at $37^{\circ} \mathrm{C}, 150 \mathrm{rpm}$ for 24 hours. Then the sample was analysed by using UV-Vis spectrometry for the confirmation of silver nanoparticles synthesis. Further SEM was used for the detection of shape of the synthesized nanoparticles.

\section{Characterization of silver nanoparticles}

UV-Vis spectroscopy: Silver nanoparticles exhibits yellowish brown colour in aqueous solution due to the phenomenon of Surface Plasmon Resonance. Thus silver nanoparticles formed were separated from the residual and characterized by UV-VIS absorption spectroscopy. Bioreduction of pure silver ion was monitored. Deionised water was used as a blank. The wavelength range for the silver nanoparticles detection was 300 to $700 \mathrm{~nm}$ and the presence of reduced silver ions was highlighted by a peak of absorption in the range of 350 to $500 \mathrm{~nm}$.

Scanning electron microscope (SEM) analysis: SEM analysis was done by using Hitachi $3700 \mathrm{~N}$ SEM machine. A thin films of sample was prepared on a carbon coated copper grid by dropping very small amount of sample on the grid, extra solution was removed by using blotting paper and then film on the SEM grid was allowed to dry by putting it under a mercury lamp for 5 minutes.

\section{Results and discussion}

\section{Analysis of phytochemicals}

Qualitative analysis of phytochemicals in Plumbago zeylanica revealed the presence of terpenoid, saponin, steroid, flavonoid, saponin and alkaloid. These photochemical compounds are major compounds which impart medicinal value of this plant. Dhale \& Markandeva ${ }^{5}$ a reported the presence of glycoside, alkaloids, reducing sugar, tannins, flavonoids and saponins in the leaves of Plumbago zeylanica. Borhade et al. ${ }^{6}$ also reported similar kinds of results. Tyagi \& Menghani ${ }^{7}$ also reported that the presence of reducing sugars, flavanoids, alkaloids and terpenoids and absence of tannins in aqueous extract of $P$. zeylanica (Table 1).

\section{Silver nanoparticles synthesis}

Aqueous extract of Plumbago zeylanica were tested for their potential to form silver nano-particle by using aqueous extract of 
whole plant. Change in the colour of solution from slightly yellow to dark brown after overnight incubation the solution of silver nitrate confirms the potential of extract of the plant to form silver nanoparticles. Characterisation of silver nanoparticle was done by using UV-Vis spectrophotometer and Scanning electron microscopy (SEM) (Figure 1).

Table I Details of presence and absence of phyto-chemicals in P. Zeylanica

\begin{tabular}{|c|c|c|}
\hline Phytochemicals & Result & Observation \\
\hline Steroid & + & Formation of Reddish Brown Ring \\
\hline Reducing Sugars & + & Reddish Brown Color \\
\hline Flavanoids & + & $\begin{array}{l}\text { Color Changes from Light Yellow } \\
\text { Color to Colorless }\end{array}$ \\
\hline Alkaloids & + & Formation of Turbid Solution \\
\hline Tannins & - & No Colour Change \\
\hline Terpenoids & + & Formation of Reddish Brown Ring \\
\hline Saponin & + & Formation of Froth \\
\hline
\end{tabular}

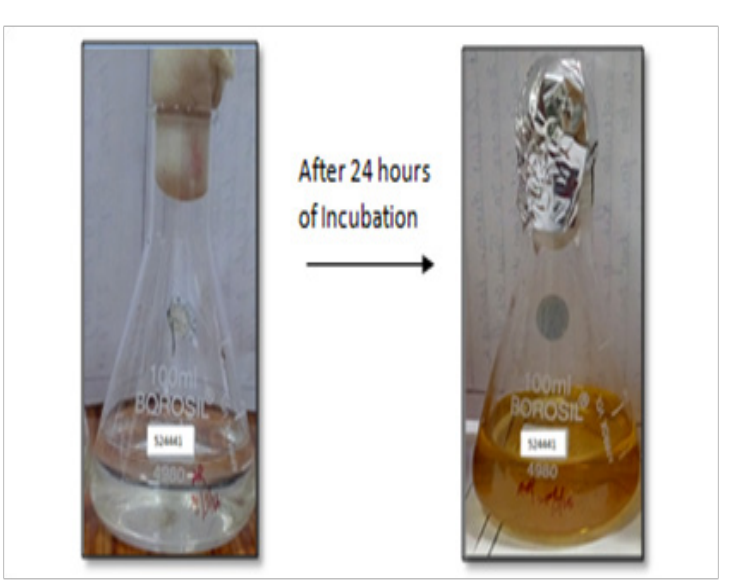

Figure I Synthesis of silver nanoparticles using plant extract

\section{UV-Vis spectroscopy}

UV-Vis analysis was done to check the formation of silver nanoparticles based on the absorbance at 350 to $450 \mathrm{~nm}$ which indicates the presence of silver nanoparticles. Peak of silver nanoparticles was found approximately at $446 \mathrm{~nm}$ at absorbance of 1.97 which corresponds to Plasmon excitation of silver nanoparticles (Figure 2). Broadening of peak indicates that the particles are poly dispersed. Renisheya Joy Jeba Malar \& Johnson ${ }^{8}$ also characterised the silver nanoparticles prepared from leaf extract of $P$. zeylanica by using $\mathrm{UV}-\mathrm{Vis}$. They have also reported the absorbance lying at the range of 430 to $470 \mathrm{~nm}$. Salunke et al. ${ }^{3}$ also reported that absorbance of silver nanoparticles was maximum at $440 \mathrm{~nm}$. Ponarulselvam et al. ${ }^{9}$ reported the absorbance of synthesized silver nanoparticles from C. roreus was maximum at $400 \mathrm{~nm}$. It was observed that nanoparticles solution was stable for more than six months with little signs of aggregation. ${ }^{10}$

\section{Scanning electron microscope (SEM)}

Scanning Electron Microscope (SEM) Analysis SEM provides the details about the morphology of the silver nanoparticles. SEM image was taken at $500 \mathrm{~nm}$. Size of synthesized nanoparticles was more than the size of nanoparticles which should be between 1-100nm. Size was more than the desired size due to the protein which were bound in the surface of nanoparticles. Result shows that the particles were of relatively spherical in shape (Figure 3).

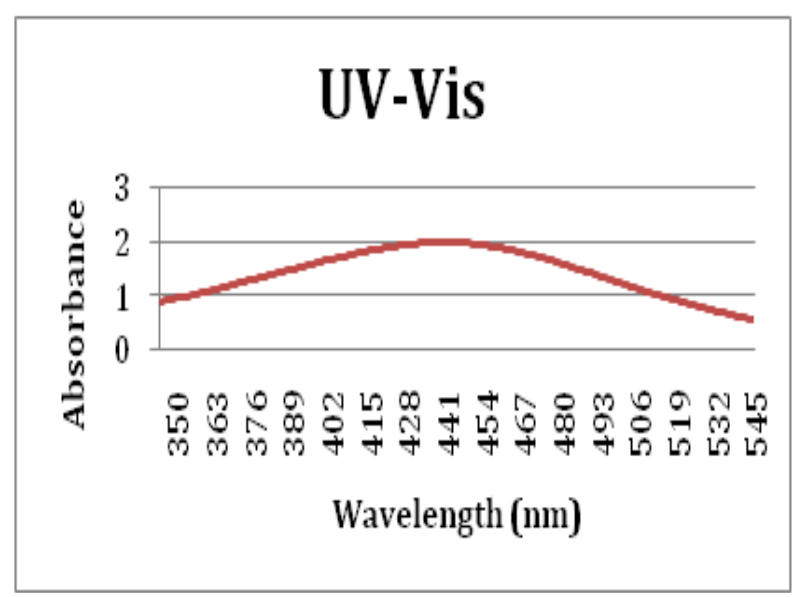

Figure 2 UV-Vis spectra of synthesized silver nanoparticles.

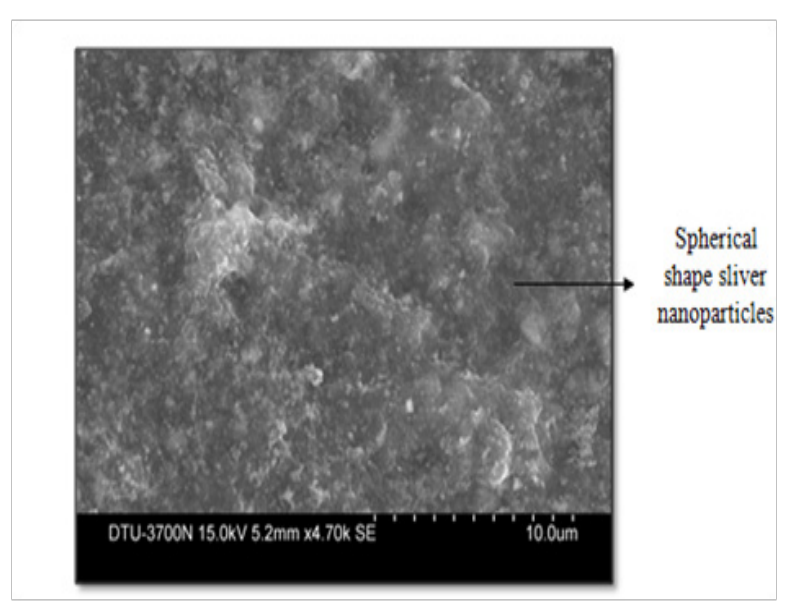

Figure 3 SEM image of silver nanoparticles.

\section{Conclusion}

Qualitative analysis of phytochemicals revealed the presence of steroids, terpenoids, saponin flavonoids, alkaloid and reducing sugar in Plumbago zeylanica. Presence of these compounds suggests the medicinal properties of this plant. There is a need of further studies to know their biological effects which could be beneficial in the treatment of various diseases.

Biosynthesis of silver nanoparticles using Plumbago zeylanica aqueous extract provides a simple, efficient and environment friendly method for the synthesis of nanoparticles. UV-VIS showed that maximum peak was obtained at $446 \mathrm{~nm}$. SEM image showed that the synthesized silver nanoparticles were relatively spherical in shape. These silver nanoparticles have potential application in biomedical field; they have several advantages such as cost effective, compatible for medical and pharmaceutical applications.

\section{Acknowledgements}

Sincere thanks to the Department of Biotechnology, Delhi Technological University for making necessary facilities during this 
study, National Bureau of Plant Genetic Resources, New Delhi for providing the plant materials and Department of Applied Physics, Delhi Technological University for providing SEM facility.

\section{Conflict of interest}

The author declares no conflict of interest.

\section{References}

1. Yuvaraj D Mandavkar, Sunil S Jalalpure. A comprehensive review on Plumbago zeylanica Linn. African journal of Pharmacy and Pharmacology. 2011;5(25):2738-2747.

2. Kumar R, Kumar S, Patra A, et al. Hepatoprotective activity of aeria parts of Plumbago zeylanica linn against carbon tetrachloride-induced hepatotoxicity in rats. International Journal of Pharmacy and Pharmaceutical Sciences. 2009;1(Suppl 1):171-175.

3. Salunke GR, Ghosh S, Santosh Kumar RJ, et al. Rapid efficient synthesis and characterization of silver, gold, and bimetallic nanoparticles from the medicinal plant Plumbago zeylanica and their application in biofilm control. Int J Nanomedicine. 2014;9:2635-2653.

4. Logeswari P, Silaqmbarasan S, Abraham J. Eco-friendly synthesis of silver nanoparticles from commercially available plant powders and their antibacterial properties. Scientia Iranica. 2013;20(3):1049-1054.
5. Dhale DA, Markandeya SK. Antimicrobial and phytochemical screening of Plumbago zeylanica Linn (plumbaginaceae) leaf. Journal of Experimental Science. 2011:2(3).

6. Borhade PS, Deshmukh TA, Patil VR, et al. Pharmacognostic and Phytochemical Investigations of Plumbago zeylanica Linn, root. Journal of Pharmacognosy and Phytochemistry. 2014;2(6):83-88.

7. Tyagi R, Menghani E. Phytochemical screening of Plumbago zeylanica: A potent Herb. International Journal of Pharma Sciences and Research. 2014;5(3):71-72.

8. Renisheya Joy Jeba Malar T, Johnson M. Biosynthesis of Silver nanoparticles using Plumbago zeylanica leaves extracts and their bio-efficacy. Research in Pharmacy. 2015;5:49-53.

9. Ponarulselvam S, Panneerselvam C, Murugan K, et al. Synthesis of silver nanoparticles using leaves of Catharanthus roseus Linn. G. Don and their antiplasmodial activities. Asian Pac J Trop Biomed. 2012;2(7):574-580.

10. Saifuddin N, Wong CW, Nur Yasumira AA. Rapid biosynthesis of silver nanoparticles using culture supernatant of bacteria with microwave irradiation. E Journal of Chemistry. 2009;6(1):61-70. 